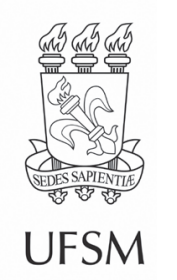

\title{
Artigos
}

\section{Regeneração natural após 13 anos de plantio com espécies arbóreas fixadoras de nitrogênio em Valença, no estado do Rio de Janeiro}

\author{
Natural regeneration after 13 years of revegetation with nitrogen- fixing \\ trees in Valença, Rio de Janeiro state, Brazil
}

\author{
Khadidja Dantas Rocha de Lima ${ }^{\oplus}$ \\ Maria Elizabeth Fernandes Correia" $\odot$ \\ Eduardo Francia Carneiro Campello" $\odot$ \\ Alexander Silva de Resende ${ }^{\prime \prime} \odot$
}

'Escola da Assembleia Legislativa do Rio Grande do Norte, Natal, RN, Brasil

"Centro Nacional de Pesquisa de Agrobiologia, Seropédica, RJ, Brasil

\section{RESUMO}

Este trabalho avaliou a regeneração natural em área plantada há 13 anos com diferentes proporções de leguminosas fixadoras de nitrogênio. O plantio foi realizado em 2001, em pastagem abandonada, em Valença, sul do estado do Rio de Janeiro, com seis espécies florestais arbóreas não nodulantes, consorciadas com sete espécies leguminosas arbóreas nodulantes, de forma a estabelecer quatro proporções de leguminosas (30, 50, 65 e 80\%) na composição botânica inicial. O delineamento experimental foi o de blocos ao acaso, com três repetições em parcelas de $940 \mathrm{~m}^{2}$. Em 2014, foram avaliados indivíduos regenerantes $(\geq 60 \mathrm{~cm}$ ) dentro de cada parcela e as espécies foram identificadas e classificadas quanto ao hábito de crescimento, grupo ecológico e síndrome de dispersão. Quantificouse densidade absoluta, frequências absoluta e relativa e os índices de diversidade de Shannon e de equabilidade de Pielou. Foram encontradas 37 espécies que não haviam sido plantadas inicialmente. Somente três, das 13 espécies plantadas estiveram presentes no banco de plântulas. A maioria das espécies regenerantes eram arbóreas, pioneiras, com hábito de dispersão por zoocoria. O plantio misto com leguminosas arbóreas nodulantes é eficaz na ativação do processo de sucessão ecológica, uma vez que garante um alto recrutamento de espécies nativas regenerantes, com destaque para a composição botânica com 50 e 65\%.

Palavras-chave: Leguminosas arbóreas; Recuperação de áreas degradadas; Floresta estacional semidecidual 


\section{ABSTRACT}

This study aimed to evaluate the ecological succession under different proportions of nitrogen-fixing trees intercropped with non-nitrogen fixing trees species through the understory floristic survey. A randomized block design with four treatments and three replications was conducted for 13 years with $30,50,65$, and $80 \%$ of nitrogen-fixing trees in the whole plants used per plot. Fixed subplots with 10 $\mathrm{m} \times 10 \mathrm{~m}$ were installed at the experimental plots and evaluated all individuals with height $\geq 60 \mathrm{~cm}$ to carry out the floristic survey. The species were identified and classified according to growth habit, the successional class, and dispersion syndrome. The natural regeneration vertical structure was evaluated with the following indicators: absolute density, absolute frequency, and relative frequency, the horizontal structure with Shannon's diversity index and Pielou's equitability index. The survey founded 37 species that had not been planted initially. Only three of the 13 planted species were present in the seedling bank. It was identified a more significant presence of pioneer trees species with zoochoric dispersion in the four treatments understory, indicating that the species mixed allowed the activation of the natural succession process. Treatments with 50 and $65 \%$ of nitrogen-fixing trees favoured the diversity of species.

Keywords: Leguminous trees; Land reclamation; Semideciduous seasonal forest

\section{INTRODUÇÃO}

No Vale do rio Paraíba do Sul, localizado ao sul do estado do Rio de Janeiro, a Mata Atlântica sofreu grandes impactos durante diferentes ciclos econômicos. No século XIX, a economia da região foi impulsionada pelo ciclo do café, em que extensas áreas foram desmatadas. Com a decadência da cafeicultura, a paisagem cedeu lugar às pastagens e fragmentos florestais em diferentes níveis de degradação e sucessão ecológica (SANTOS et al., 2009).

Para reverter essa degradação, são necessárias iniciativas de restauração ecológica (MARTIN, 2017). Nesse contexto, o plantio de espécies arbóreas visa acelerar o processo de restauração e, para seu sucesso, a escolha das espécies é de extrema importância (RESENDE; LELES, 2017). As espécies escolhidas para recompor essas áreas devem conseguir se estabelecer em ambiente degradado, superar a competição com gramíneas exóticas (SANTANA et al., 2020) e facilitar a entrada de novas espécies, aumentando a complexidade da comunidade (KELTY, 2006). 
Nesse contexto, leguminosas arbóreas que formam associações simbióticas com bactérias capazes de fixar nitrogênio atmosférico e com fungos micorrízicos, podem ser indicadas como facilitadoras do processo sucessional, por apresentarem: (i) maior rusticidade na competição por água, nutrientes e energia, (ii) rápido crescimento e (iii) por promoverem condições favoráveis à entrada e permanência de indivíduos mais exigentes (MACHADO et al., 2010). Este último está relacionado ao aumento das condições de sombreamento, aporte de nutrientes (via deposição de serapilheira) e transferência de nitrogênio para as espécies coabitantes (via rede micelial de fungos micorrízicos). Todas essas ações são esperadas, podendo levar à redução de custos e aumento da eficiência dos processos de recuperação dessas áreas (MARTINS et al., 2019).

Diversos estudos têm avaliado o efeito de plantios consorciados, em que são testados os efeitos de uma ou mais espécies de leguminosas arbóreas sobre outras não nodulantes (SILVA et al., 2013; LIMA et al., 2017). Interferências por vezes positivas e negativas na qualidade do solo e no desenvolvimento das espécies consorciadas já foram observadas. Entretanto, pouco se sabe sobre as interações ocorridas nesses modelos de plantios sobre o processo de sucessão ecológica.

Dessa maneira, o objetivo deste trabalho foi o de avaliar o efeito do plantio de diferentes proporções de leguminosas arbóreas fixadoras de nitrogênio, em consórcio com espécies arbóreas não nodulantes, sobre a sucessão ecológica, no bioma Mata Atlântica.

\section{MATERIAL E MÉTODO}

O estudo foi desenvolvido na Fazenda Santo Antônio da Aliança, localizada no Santuário de Vida Silvestre da Serra da Concórdia, criado em 1992 para preservar a fauna e a flora do ecossistema da Serra da Concórdia, que é o mais importante remanescente da Mata Atlântica na margem esquerda do Médio Paraíba do Sul. Faz

parte do Projeto Santuários de Vida Silvestre da Fundação Pró-Natureza - Funatura. É 
uma APA (Área de Proteção Ambiental) e, portanto, não é uma unidade de proteção integral, o que possibilita alguns usos diretos e indiretos. Localiza-se na divisa dos municípios de Barra do Piraí e Valença, Rio de Janeiro, situado entre as coordenadas geográficas de $22^{\circ} 22^{\prime} 20^{\prime \prime} \mathrm{S}$ e 4347'23"W, com altitude média de 650 m (CALDAS, 2006).

Pela classificação de Köppen, o clima da região é Cwa, tropical de altitude, com precipitação pluviométrica de $1.225 \mathrm{~mm}$, temperatura média das máximas de $30^{\circ} \mathrm{C}$ e umidade relativa do ar de $72 \%$ (DERESZ et al., 2006). O relevo da área é predominantemente ondulado, característico da região sob influência do domínio morfoclimático dos “mares-de-morros” (AB'SABER, 1966). Os solos mais representativos da área são o Cambissolo Háplico Tb distrófico e o Latossolo Vermelho-Amarelo distrófico (CALDAS, 2006).

A área experimental está inserida na região fitoecológica denominada Floresta Estacional Semidecidual, que apresenta deficit hídrico durante a estação seca, ocasionando perda das folhas pelas espécies arbóreas nessa estação (IBGE, 1992). Em março de 2001, foi realizado o reflorestamento de uma área de pasto abandonado, a partir do plantio de mudas de seis espécies florestais arbóreas não nodulantes, consorciadas com mudas de sete espécies leguminosas arbóreas nodulantes (Tabela 1), plantadas com o objetivo de facilitar o estabelecimento das demais. Foram usadas espécies nativas, misturadas com espécies não nativas (Khaya senegalensis nativa da África, Acacia auriculiformis nativa da Austrália, Acacia mangium nativa da Austrália e Pseudosamanea guachapele nativa da América Central). No plantio houve a preocupação de mesclar espécies com potencial econômico e outras de elevada plasticidade ambiental, junto com nativas, de forma a garantir o sucesso do plantio e atender à demanda do proprietário de possibilitar renda à restauração florestal de sua área. Realidade que se aplica aos dias de hoje, no que tange à recomposição de reserva legal. Outra característica desse estudo, diz respeito à avaliação da presença de espécies exóticas na composição botânica do plantio e sua possível interferência na regeneração natural. Questão de elevada preocupação nos dias atuais e relativizada quando do plantio da área em 2001. 
O delineamento experimental utilizado foi em blocos ao acaso, com quatro tratamentos, considerando as proporções de leguminosas arbóreas nodulantes distribuídas em 30, 50, 65 e 80\% do total de plantas de cada unidade experimental. Todos os tratamentos foram repetidos em três blocos. Totalizando 12 parcelas, cada uma com dimensões de 37,5 m x 25 m.

Tabela 1 - Espécies arbóreas fixadoras e não fixadoras de nitrogênio utilizadas no experimento em Valença, estado do Rio de Janeiro

\begin{tabular}{|c|c|c|}
\hline Nome científico & Nome vulgar & Família \\
\hline \multicolumn{3}{|c|}{ Espécies não nodulantes } \\
\hline Ceiba speciosa (A. St. Hil.) Ravenna & Paineira & Malvaceae \\
\hline Colubrina glandulosa Perkins & Sobrasil & Rhamnaceae \\
\hline Handroanthus chrysotrichus (Mart. ex DC.) Mattos & Ipê-amarelo & Bignoniaceae \\
\hline Khaya senegalensis (Desr.) A. Juss & Mogno-africano & Meliaceae \\
\hline Peltophorum dubium (Sprengel) Taub & Farinha-seca & $\begin{array}{c}\text { Fabaceae- } \\
\text { Caesalpinoideae }\end{array}$ \\
\hline Handroanthus heptaphyllus (Vell.) Mattos & Ipê-rosa & Bignoniaceae \\
\hline \multicolumn{3}{|c|}{ Espécies nodulantes Fabaceae } \\
\hline Acacia auriculiformis A. Cunn. ex. Benth. & Acácia-auriculada & Mimosoideae \\
\hline Acacia mangium Willd. & Acácia-mangium & Mimosoideae \\
\hline Dalbergia nigra (Vell.) Alemão ex. Benth. & Jacarandá-da-bahia & Papilionoideae \\
\hline Enterolobium contortisiliquum (Vell.) Morong. & Orelha-de-macaco & Mimosoideae \\
\hline Mimosa caesalpiniifolia Benth. & Sabiá & Mimosoideae \\
\hline Piptadenia gonoacantha (Mart.) J. F. Macbr. & Pau-jacaré & Mimosoideae \\
\hline Pseudosamanea guachapele (Kunth) Harms. & Guachapele & Mimosoideae \\
\hline
\end{tabular}

Fonte: Autores (2020)

As mudas foram produzidas em viveiro e as sementes das espécies de leguminosas nodulantes foram inoculadas com estirpes de rizóbios e propágulos de fungos micorrízicos arbusculares (FMA), conforme recomendações de Resende et al. (2013). Já as espécies arbóreas não nodulantes foram inoculadas apenas com FMA. Todos os inoculantes foram produzidos e fornecidos pela Embrapa Agrobiologia. 
O plantio foi realizado em covas de 0,2 $\mathrm{m} \times 0,2 \mathrm{~m} \times 0,2 \mathrm{~m}$, adubadas com $100 \mathrm{~g}$ de fosfato de rocha $+10 \mathrm{~g}$ de composto de micronutrientes (12\% de $\mathrm{Zn}, 1,6 \%$ de $\mathrm{Cu}$, 4\% de Mn e 1,8\% de B) + 25 g de sulfato de potássio + 25 g de calcário dolomítico. Cada parcela foi composta por 204 mudas, em espaçamento de $2 \mathrm{~m} \times 2 \mathrm{~m}$, totalizando 2.448 mudas, plantadas em curvas de nível.

Até o fechamento da área, aos quatro anos, foram realizadas dez atividades de roçada e coroamento além do replantio das mudas mortas na área experimental. Alves (2007) avaliou a taxa de sobrevivência das espécies não nodulantes aos 48 meses após o plantio (Tabela 2). Nota-se que as diferentes proporções de leguminosas no stand não afetou de forma consistente a média da taxa de sobrevivência que ficou próxima a 40\%. As espécies C. speciosa e $H$. heptaphyllus não foram plantadas no tratamento de $65 \%$ de leguminosas, por problemas no processo de produção de mudas. Dessa forma, em substituição, aumentou-se o número de indivíduos das outras 4 espécies. C. speciosa e P. dubium apresentaram as maiores taxas de sobrevivência.

Tabela 2 - Taxa de sobrevivência das espécies não fixadoras de nitrogênio 48 meses após o plantio, em função das proporções de leguminosas utilizadas

\begin{tabular}{|c|c|c|c|c|}
\hline \multirow[t]{2}{*}{ Nome científico } & \multicolumn{4}{|c|}{$\begin{array}{l}\text { Taxa de sobrevivência em função do } \\
\text { percentual de leguminosas plantadas (\%) }\end{array}$} \\
\hline & 30 & 50 & 65 & 80 \\
\hline Ceiba speciosa (A. St. Hil.) Ravenna & 67 & 75 & - & 56 \\
\hline Colubrina glandulosa Perkins & 37 & 30 & 33 & 8 \\
\hline Handroanthus chrysotrichus (Mart. ex DC.) Mattos & 26 & 35 & 42 & 29 \\
\hline Khaya senegalensis (Desr.) A. Juss & 32 & 10 & 20 & 0 \\
\hline Peltophorum dubium (Sprengel) Taub & 81 & 71 & 73 & 86 \\
\hline Handroanthus heptaphyllus (Vell.) Mattos & 40 & 21 & - & 26 \\
\hline Média & 47 & 40 & 42 & 42 \\
\hline
\end{tabular}

Fonte: Adaptado de Alves (2007)

Em outubro de 2014, final do período seco, foi realizado um levantamento florístico e fitossociológico das espécies regenerantes do sub-bosque, em que foram demarcadas subparcelas de $10 \mathrm{~m} \times 10 \mathrm{~m}$, em cada uma das 12 parcelas experimentais. 
A identificação botânica das espécies foi realizada in loco, partindo do reconhecimento de suas estruturas vegetativas (folhas) e reprodutivas (flor, fruto e sementes). Para as espécies não identificadas em campo, foi necessária a coleta do material botânico fértil, quando possível, para identificação através da confecção de exsicatas. O material vegetal coletado foi identificado com base no acervo da coleção botânica do Herbário do Jardim Botânico do Rio de Janeiro (REFLORA, 2015). As espécies foram classificadas quanto ao hábito de crescimento (arbóreo, arbustivo e herbáceo), ao grupo ecológico (pioneira e não pioneira) e à síndrome de dispersão (anemocórica, zoocórica e autocórica) segundo a lista indicada pela Resolução SMA n 08/2008 do Instituto de Botânica de São Paulo.

Os parâmetros fitossociológicos considerados para a análise da estrutura horizontal estão descritos em Freitas e Magalhães (2012) e abrangeram as Densidades Absoluta (DA) e as Frequências Absoluta (FA) e Relativa (FR). A diversidade florística foi estimada pelo índice de diversidade de Shannon $\left(\mathrm{H}^{\prime}\right)$ e a equabilidade pelo índice de Pielou ( $\left.J^{\prime}\right)$.

Os dados do levantamento florístico (família botânica, gêneros, espécies) foram tratados por meio de estatística descritiva (valores mínimos, máximos, médias e coeficientes de variação).

Os possíveis padrões de relação entre a porcentagem de leguminosas arbóreas nodulantes plantadas e a regeneração natural no sub-bosque das áreas estudadas foram verificados através de uma análise multivariada de agrupamento (cluster analysis). Dessa maneira, obteve-se um dendrograma de distâncias pelo método da ligação simples (single linkage method) e distância euclidiana, por meio do cálculo da média dos valores de equabilidade e diversidade, para cada tratamento, com o auxílio do programa PAST versão 2.17c. Essa análise permite agrupar áreas consideradas semelhantes.

\section{RESULTADOS E DISCUSSÃO}

As diferentes proporções de leguminosas influenciaram no número total de indivíduos regenerantes. O menor valor médio foi encontrado no tratamento de 80\% 
de leguminosas (8.300 ind.ha ${ }^{-1}$ ) e o maior, no tratamento de 65\% (11.367 ind.ha-1) (Tabela 3). Já em relação ao número de famílias, gêneros e espécies de regenerantes associados a cada tratamento, os menores valores de riqueza de famílias (12), gêneros (17) e espécies (19), estiveram associados ao tratamento de 30\% de leguminosas na composição de plantio. As demais proporções de leguminosas apresentaram semelhanças entre si, com valores médios de 19 famílias, 26 gêneros e 28 espécies. Em média, os tratamentos com percentuais intermediários de leguminosas favoreceram em mais de $60 \%$ a regeneração natural da área, quando comparados ao tratamento com menor percentual de leguminosas na composição botânica, após 13 anos do plantio.

Tabela 3 - Densidade total de indivíduos, número de famílias, gêneros e espécies, dos indivíduos regenerantes no sub-bosque, em Valença, estado do Rio de Janeiro

\begin{tabular}{|c|c|c|c|c|c|}
\hline Tratamentos & Mínimo & Média & Máximo & Total & CV (\%) \\
\hline \multicolumn{6}{|c|}{ Densidade total de indivíduos (indivíduos ha-1) } \\
\hline $30 \%$ & 8.100 & 9.300 & 10.400 & - & 12 \\
\hline $50 \%$ & 8.000 & 11.000 & 12.900 & - & 24 \\
\hline $65 \%$ & 10.500 & 11.367 & 12.700 & - & 10 \\
\hline $80 \%$ & 7.000 & 8.300 & 9.800 & - & 17 \\
\hline \multicolumn{6}{|c|}{ Famílias (número) } \\
\hline $30 \%$ & 6 & 8 & 10 & 12 & 25 \\
\hline $50 \%$ & 8 & 12 & 15 & 19 & 30 \\
\hline $65 \%$ & 10 & 11 & 12 & 16 & 9 \\
\hline $80 \%$ & 8 & 12 & 19 & 21 & 51 \\
\hline \multicolumn{6}{|c|}{ Gêneros (número) } \\
\hline $30 \%$ & 7 & 10 & 11 & 17 & 24 \\
\hline $50 \%$ & 10 & 14 & 20 & 27 & 36 \\
\hline $65 \%$ & 13 & 14 & 15 & 25 & 8 \\
\hline $80 \%$ & 10 & 14 & 23 & 24 & 52 \\
\hline \multicolumn{6}{|c|}{ Espécies (número) } \\
\hline $30 \%$ & 9 & 11 & 13 & 19 & 18 \\
\hline $50 \%$ & 11 & 16 & 21 & 29 & 32 \\
\hline $65 \%$ & 14 & 16 & 18 & 28 & 13 \\
\hline $80 \%$ & 10 & 15 & 24 & 26 & 52 \\
\hline
\end{tabular}

Fonte: Autores (2020) 
Esses resultados reforçam o papel facilitador que as espécies fixadoras de nitrogênio possuem, uma vez que, como apontado por Chaer et al. (2011) e Menge e Chazdon (2016), ao melhorarem as condições de disponibilidade de nitrogênio do substrato, permitem que mais espécies possam ocupar esses nichos, estruturando o ambiente.

De modo geral, a família Fabaceae, com 9 espécies, apresentou a maior riqueza (Tabela 4). Avaliando fragmentos limítrofes a essa área experimental, Freitas e Magalhães (2014) também encontraram maior riqueza de espécies dessa família. Esses resultados são comuns em regiões tropicais que apresentam uma estação seca prolongada. Segundo Ribeiro et al. (2009), isso se dá em função de algumas espécies dessa família formarem associações simbióticas com fungos micorrízicos e rizóbios, acarretando vantagem competitiva por água, energia e nutrientes, em comparação com famílias que não fazem uso dessa dupla associação.

De maneira geral, 10 espécies foram as responsáveis por $73 \%$ de todos os indivíduos encontrados na regeneração natural (Tabela 4), ficando as outras 30 espécies com os $27 \%$ restantes. Outro ponto a ser destacado é que, embora tenham sido plantadas 7 espécies da família Fabaceae, somente três delas foram encontradas no banco de plântulas regenerantes (Piptadenia gonoacantha $(7,4 \%)$ (nativa da região), Mimosa caesalpiniifolia (2,1\%) e Pseudosamanea guachapele (0,4\%)(ambas exóticas à região), totalizando $10 \%$ dos indivíduos. Com exceção dessas duas últimas, nenhuma outra espécie não nativa do bioma, que havia sido plantada inicialmente, foi encontrada no banco de plântulas. Todas as demais foram provenientes da regeneração natural das fontes de propágulos existentes no entorno ou presentes no banco de sementes do solo.

Esse resultado reforça o papel de facilitação de espécies leguminosas pioneiras, fixadoras de nitrogênio, que estruturam o ambiente, mas não apresentam representatividade no banco de plântulas, permitindo assim que espécies nativas se estabeleçam a partir da melhoria das condições edafoclimáticas (RESENDE et al., 2013). 
O porte das plantas regenerantes do sub-bosque foi essencialmente arbóreo (acima de 70\%) para todos os tratamentos, com forte predomínio de espécies pioneiras (próximo a 80\%) e com dispersão zoocórica (acima de 55\%). A maior presença de espécies com hábito de crescimento arbóreo é um bom indicativo de favorecimento da sucessão ecológica, pois, à medida que as espécies plantadas vão entrando em senescência, as espécies arbóreas regenerantes irão substituí-las, dando sequência ao processo sucessional.

Em todos os tratamentos, as espécies pioneiras apresentaram maior frequência e dominância. A maior presença de espécies pioneiras pode estar associada a um relativo curto período entre o plantio das espécies arbóreas e a avaliação florística (13 anos), como também à ausência de fragmentos florestais em estágios avançados de sucessão ecológica ou de mata nativa limítrofe às áreas estudadas (CALDAS, 2006).

Tabela 4 - Espécies encontradas no sub-bosque dos plantios com 30, 50, 65 e 80\% de leguminosas arbóreas fixadoras de nitrogênio, em Valença, estado do Rio de Janeiro

\begin{tabular}{|c|c|c|c|c|c|c|c|c|c|c|c|c|c|c|}
\hline \multirow{3}{*}{ Família } & \multirow{3}{*}{ Nome Científico } & \multirow{3}{*}{$\mathrm{HC}$} & \multirow{3}{*}{ G } & \multirow{3}{*}{ D } & \multicolumn{8}{|c|}{ Tratamentos } & \multirow{3}{*}{ FRi } & \multirow{3}{*}{$\begin{array}{l}\text { FR } \\
\text { corrência/ } \\
\text { parcelas }\end{array}$} \\
\hline & & & & & \multicolumn{2}{|c|}{30} & \multicolumn{2}{|c|}{50} & \multicolumn{2}{|c|}{65} & \multicolumn{2}{|c|}{80} & & \\
\hline & & & & & DAi & FAi & DAi & FAi & DAi & FAi & DAi & FAi & & \\
\hline Anacardiaceae & $\begin{array}{c}\text { Schinus terebinthifolius } \\
\text { Raddi }\end{array}$ & Arbóreo & $P$ & Zo & - & - & 100 & 8,3 & - & - & - & - & 0,2 & 8,3 \\
\hline Annonaceae & Annona cacans Warm. & Arbóreo & $\mathrm{P}$ & Zo & - & - & 200 & 8,3 & - & - & 100 & 8,3 & 0,6 & 16,7 \\
\hline Arecaceae & $\begin{array}{c}\text { Syagrus romanzoffiana } \\
\text { (Cham.) Glassman }\end{array}$ & Arbóreo & $P$ & Zo & 100 & 8,3 & 800 & 25,0 & 1200 & 25,0 & 1600 & 25,0 & 7,2 & 83,3 \\
\hline \multirow{3}{*}{ Asteraceae } & $\begin{array}{c}\text { Piptocarpha axillaris } \\
\text { (Less.) Baker }\end{array}$ & Arbóreo & $P$ & An & - & - & - & - & - & - & 100 & 8,3 & 0,2 & 8,3 \\
\hline & Vernonanthura & & & & & & & & & & & & & \\
\hline & $\begin{array}{c}\text { phosphorica (Vell.) H. } \\
\text { Rob. }\end{array}$ & Arbustivo & $\mathrm{P}$ & An & - & - & 200 & 8,3 & - & - & - & - & 0,4 & 8,3 \\
\hline Bignoniaceae & $\begin{array}{l}\text { Cybistax antisyphilitica } \\
\text { (Mart.) Mart. }\end{array}$ & Arbóreo & NP & An & 200 & 16,7 & 100 & 8,3 & 500 & 16,7 & 300 & 25,0 & 2,1 & 66,7 \\
\hline Cecropiaceae & Cecropia hololeuca Miq. & Arbóreo & $P$ & Zo & - & - & 300 & 16,7 & 100 & 8,3 & 100 & 8,3 & 1,0 & 33,3 \\
\hline Euphorbiaceae & $\begin{array}{c}\text { Alchornia glandulosa } \\
\text { Poepp. \& Endl. }\end{array}$ & Arbóreo & $P$ & Zo & - & - & 100 & 8,3 & - & - & 100 & 8,3 & 0,4 & 16,7 \\
\hline $\begin{array}{l}\text { Fabaceae- } \\
\text { Caesalpinioideae }\end{array}$ & $\begin{array}{l}\text { Senna pendula (Humb. } \\
\text { Bonpl. ex Willd.) H. S. } \\
\text { Irwin Barneby. }\end{array}$ & Arbóreo & $P$ & Zo & 100 & 8,3 & 100 & 8,3 & - & - & 300 & 8,3 & 0,8 & 33,3 \\
\hline
\end{tabular}


Tabela 4 - Continuação

\begin{tabular}{|c|c|c|c|c|c|c|c|c|c|c|c|c|c|c|}
\hline \multirow{3}{*}{ Família } & \multirow{3}{*}{ Nome Científico } & \multirow{3}{*}{$\mathrm{HC}$} & \multirow{3}{*}{$\mathbf{G}$} & \multirow{3}{*}{ D } & \multicolumn{8}{|c|}{ Tratamentos } & \multirow{3}{*}{ FRi } & \multirow{3}{*}{$\begin{array}{c}\text { FR } \\
\text { Ocorrência/ } \\
\text { parcelas }\end{array}$} \\
\hline & & & & & \multicolumn{2}{|c|}{30} & \multicolumn{2}{|c|}{50} & \multicolumn{2}{|c|}{65} & \multicolumn{2}{|c|}{80} & & \\
\hline & & & & & DAi & $F A i$ & DAi & $\mathbf{F A i}$ & DAi & $F A i$ & DAi & FAi & & \\
\hline \multirow{6}{*}{$\begin{array}{l}\text { Fabaceae- } \\
\text { Papilionoideae }\end{array}$} & Centrolobium & & & & & & & & & & & & & \\
\hline & $\begin{array}{c}\text { microchaete (Mart. ex } \\
\text { Benth.) H. C. Lima }\end{array}$ & Arbóreo & NP & An & - & - & - & - & 100 & 8,3 & 100 & 8,3 & 0,4 & 16,7 \\
\hline & $\begin{array}{l}\text { Machaerium nyctitans } \\
\text { (Vell.) Benth. }\end{array}$ & Arbóreo & $\mathrm{P}$ & An & - & - & - & - & 100 & 8,3 & - & - & 0,2 & 8,3 \\
\hline & $\begin{array}{c}\text { Anadenanthera } \\
\text { colubrina (Vell.) Brenan }\end{array}$ & Arbóreo & $\mathrm{P}$ & $\mathrm{Au}$ & - & - & - & - & 100 & 8,3 & - & - & 0,2 & 8,3 \\
\hline & $\begin{array}{c}\text { Mimosa artemisiana } \\
\text { Heringer \& Paula }\end{array}$ & Arbóreo & $P$ & $\mathrm{Au}$ & - & - & - & - & 300 & 16,7 & - & - & 0,6 & 16,7 \\
\hline & $\begin{array}{c}\text { Mimosa caesalpiniifolia } \\
\text { Benth. }\end{array}$ & Arbóreo & $\mathrm{P}$ & $\mathrm{Au}$ & - & - & 100 & 8,3 & 1000 & 16,7 & - & - & 2,1 & 25,0 \\
\hline \multirow[t]{4}{*}{ Mimosoideae } & $\begin{array}{l}\text { Piptadenia gonoacantha } \\
\text { (Mart.) Macbr. } \\
\text { Pseudosamanea }\end{array}$ & Arbóreo & $\mathrm{P}$ & $\mathrm{Au}$ & 600 & 16,7 & 1400 & 25,0 & 600 & 16,7 & 1200 & 25,0 & 7,4 & 83,3 \\
\hline & guachapele (Kunth) & Arbóreo & $\mathrm{P}$ & $\mathrm{Au}$ & - & - & 100 & 8,3 & 100 & 8,3 & - & - & 0,4 & 16,7 \\
\hline & Harms & & & & & & & & & & & & & \\
\hline & $\begin{array}{l}\text { Senegalia polyphylla } \\
\text { (DC.) Britton \& Rose }\end{array}$ & Arbóreo & $P$ & $\mathrm{Au}$ & 200 & 16,7 & - & - & - & - & 2800 & 25,0 & 7,0 & 50,0 \\
\hline Lauraceae & $\begin{array}{l}\text { Ocotea pulchella (Nees } \\
\qquad \& \text { Mart.) Mez }\end{array}$ & Arbóreo & $\mathrm{P}$ & Zo & - & - & - & - & - & - & 100 & 8,3 & 0,2 & 8,3 \\
\hline Malvaceae & Sida rhombifolia L. & Arbustivo & $\mathrm{P}$ & $\mathrm{Au}$ & 1000 & 16,7 & 800 & 16,7 & 1700 & 25,0 & 400 & 16,7 & 7,6 & 75,0 \\
\hline Melastomataceae & $\begin{array}{l}\text { Miconia pusiliiflora (DC.) } \\
\text { Naudin }\end{array}$ & Arbóreo & $\mathrm{P}$ & Zo & - & - & - & - & - & - & 100 & 8,3 & 0,2 & 8,3 \\
\hline \multirow[t]{2}{*}{ Meliaceae } & $\begin{array}{l}\text { Guarea guidonia (L.) } \\
\text { Sleumer }\end{array}$ & Arbóreo & $\mathrm{NP}$ & Zo & - & - & - & - & - & - & 200 & 16,7 & 0,6 & 8,3 \\
\hline & Melia azedarach L. & Arbóreo & $\mathrm{P}$ & Zo & - & - & 100 & 8,3 & - & - & - & - & 0,2 & 8,3 \\
\hline \multirow{2}{*}{ Myrtaceae } & Eugenia uniflora $\mathrm{L}$. & Arbustivo & $\mathrm{P}$ & Zo & 600 & 25,0 & 200 & 16,7 & 700 & 25,0 & 900 & 16,7 & 4,7 & 83,3 \\
\hline & Psidium guajava $\mathrm{L}$. & Arbóreo & $\mathrm{P}$ & $\mathrm{Au}$ & - & - & 200 & 8,3 & 100 & 8,3 & - & - & 0,2 & 8,3 \\
\hline \multirow{2}{*}{ Piperaceae } & Piper arboreum Aubl. & Arbóreo & $P$ & Zo & 2200 & 25,0 & 1200 & 25,0 & 1800 & 25,0 & 1800 & 25,0 & 13,6 & 100 \\
\hline & Piper cuyabanum C. DC. & Herbáceo & $\mathrm{P}$ & Zo & 1500 & 25,0 & 1100 & 25,0 & 1300 & 25,0 & 400 & 8,3 & 8,4 & 83,3 \\
\hline Rosaceae & $\begin{array}{c}\text { Eriobotrya japonica } \\
\text { (Thunb.) Lindl. }\end{array}$ & Arbóreo & NP & Zo & - & - & 100 & 8,3 & - & - & 100 & 8,3 & 0,4 & 16,7 \\
\hline Rubiaceae & $\begin{array}{c}\text { Psychotria } \\
\text { carthagenensis Jacq. }\end{array}$ & Arbóreo & NP & Zo & 100 & 8.3 & 900 & 33,3 & 600 & 25,0 & 1100 & 16,7 & 5,3 & 75,0 \\
\hline \multirow{2}{*}{ Rutaceae } & $\begin{array}{l}\text { Citrus aurantifolia } \\
\text { (Cristm.) Swingle }\end{array}$ & Arbóreo & $\mathrm{P}$ & Zo & - & - & - & - & 100 & 8,3 & - & - & 0,2 & 8,3 \\
\hline & $\begin{array}{l}\text { Zanthoxylum rhoifolium } \\
\text { Lam. }\end{array}$ & Arbóreo & $\mathrm{P}$ & Zo & - & - & - & - & - & - & 100 & 8,3 & 0,2 & 8,3 \\
\hline Sapindaceae & $\begin{array}{c}\text { Cupania vernalis } \\
\text { Cambess. }\end{array}$ & Arbóreo & NP & Zo & 200 & 16,7 & 100 & 8,3 & 100 & 8,3 & 100 & 8,3 & 0,8 & 33,3 \\
\hline
\end{tabular}


Tabela 4 - Conclusão

\begin{tabular}{|c|c|c|c|c|c|c|c|c|c|c|c|c|c|c|}
\hline \multirow{3}{*}{ Família } & \multirow{3}{*}{ Nome Científico } & \multirow{3}{*}{ HC } & \multirow{3}{*}{ G } & \multirow{3}{*}{ D } & \multicolumn{8}{|c|}{ Tratamentos } & \multirow{3}{*}{ FRi } & \multirow{3}{*}{$\begin{array}{l}\text { FR } \\
\text { corrência/ } \\
\text { parcelas }\end{array}$} \\
\hline & & & & & \multicolumn{2}{|c|}{30} & \multicolumn{2}{|c|}{50} & \multicolumn{2}{|c|}{65} & \multicolumn{2}{|c|}{80} & & \\
\hline & & & & & DAi & $\mathbf{F A i}$ & DAi & FAi & DAi & FAi & DAi & FAi & & \\
\hline \multirow{6}{*}{ Solanaceae } & $\begin{array}{l}\text { Cestrum intermedium } \\
\text { Sendtn. }\end{array}$ & Arbustivo & $P$ & Zo & 100 & 8,3 & 100 & 8,3 & 500 & 8,3 & 500 & 16,7 & 2,3 & 41,7 \\
\hline & Nicotiana tabacum L. & Arbustivo & $P$ & $\mathrm{Au}$ & 100 & 8,3 & 500 & 16,7 & 700 & 16,7 & 100 & 8,3 & 2,7 & 50,0 \\
\hline & $\begin{array}{c}\text { Solanum argenteum } \\
\text { Dunal }\end{array}$ & Arbóreo & $P$ & Zo & 800 & 16,7 & 700 & 25,0 & 900 & 25,0 & 400 & 16,7 & 5,5 & 83,3 \\
\hline & Solanum & & & & & & & & & & & & & \\
\hline & granulosoleprosum & Arbóreo & $P$ & Zo & 1400 & 25,0 & 100 & 8,3 & 100 & 8,3 & 100 & 8,3 & 3,3 & 50,0 \\
\hline & Dunal & & & & & & & & & & & & & \\
\hline Sterculiaceae & $\begin{array}{c}\text { Guazuma ulmifolia } \\
\text { Lamarck }\end{array}$ & Arbóreo & $P$ & Zo & 1300 & 25,0 & 1300 & 25,0 & 200 & 8,3 & 600 & 16,7 & 6,6 & 75,0 \\
\hline Styracaceae & Styrax pohlii A. DC. & Arbóreo & NP & Zo & 100 & 8,3 & 200 & 4,2 & 700 & 25,0 & 800 & 16,7 & 3,5 & 66,7 \\
\hline Ulmaceae & $\begin{array}{c}\text { Trema micrantha (L.) } \\
\text { Blume. }\end{array}$ & Arbóreo & $P$ & Zo & - & - & - & - & 100 & 8,3 & 100 & 8,3 & 0,4 & 16,7 \\
\hline \multirow[t]{2}{*}{ Verbenaceae } & $\begin{array}{l}\text { Aegiphila sellowiana } \\
\text { Cham. }\end{array}$ & Arbóreo & $P$ & Zo & - & - & 100 & 2,1 & - & - & - & - & 0,2 & 8,3 \\
\hline & Lantana camara L. & Arbustivo & $P$ & Zo & - & - & 200 & 2,1 & 200 & 8,3 & 100 & 8,3 & 1,0 & 25,0 \\
\hline
\end{tabular}

Fonte: Autores (2020)

Em que: $\mathrm{HC}=$ Hábito de Crescimento (arbóreo, arbustivo); $\mathrm{G}=$ Grupo Ecológico $(\mathrm{P}=$ Pioneira e NP = Não pioneira); $\mathrm{D}=$ Síndrome de Dispersão (Zo = Zoocórica, An = Anemocórica, Au = Autocórica), DAi = Densidade absoluta; FAi e FRi = Frequências absoluta e relativa e de ocorrência nas parcelas.

A maior presença de espécies no sub-bosque com síndrome de dispersão zoocórica sinaliza a capacidade de atratividade de fauna exercida pelas espécies plantadas. Melo e Durigan (2007) ressaltam a importância das espécies com síndrome de dispersão zoocórica, como facilitadoras do processo de sucessão, pois permitem uma atratividade à fauna e, assim, contribuem com o enriquecimento da área com novas formas de vida.

Os índices de diversidade de Shannon-Weaver $\left(\mathrm{H}^{\prime}\right)$ e de equabilidade de Pielou $\left(J^{\prime}\right)$, verificados nos tratamentos $30 \%\left(H^{\prime}=2,05\right.$ e J' $\left.=0,58\right), 50 \%\left(H^{\prime}=2,52\right.$ e J' $\left.=0,69\right), 65 \%$ $\left(H^{\prime}=2,52\right.$ e J' $\left.=0,65\right)$ e $80 \%\left(H^{\prime}=2,26\right.$ e J' = 0,59), estão representados no agrupamento 
de Cluster da Figura 1. A análise mostra alta similaridade entre os tratamentos com 50 e $65 \%$ de leguminosas plantadas no consórcio, indicando, junto com os valores absolutos de H' e J", que a regeneração natural foi favorecida nesses tratamentos.

Esses valores ainda são inferiores aos encontrados em um fragmento próximo à área de estudo, que, segundo Freitas e Magalhães (2014), foram de H'=3,15 e J' = 0,84, mas indicam que, mesmo em pouco tempo, a intervenção feita possibilitou um ganho ecológico à área. Esse fato pode ser constatado numericamente, ao se considerar que em 2001 foram plantadas 13 espécies e, em 2014, foram contabilizadas 37 espécies que não haviam sido plantadas, totalizando uma riqueza total de 50 espécies. Esses resultados reforçam o papel de estruturação ambiental que as leguminosas possuem (CHAER et al., 2011; MENGE; CHAZDON, 2016).

Figura 1 - Análise multivariada de agrupamento (cluster) por distância Euclidiana, ligação simples (single linkage) obtida a partir dos valores referentes aos índices de diversidade de Shannon e equabilidade de Pielou nos diferentes tratamentos

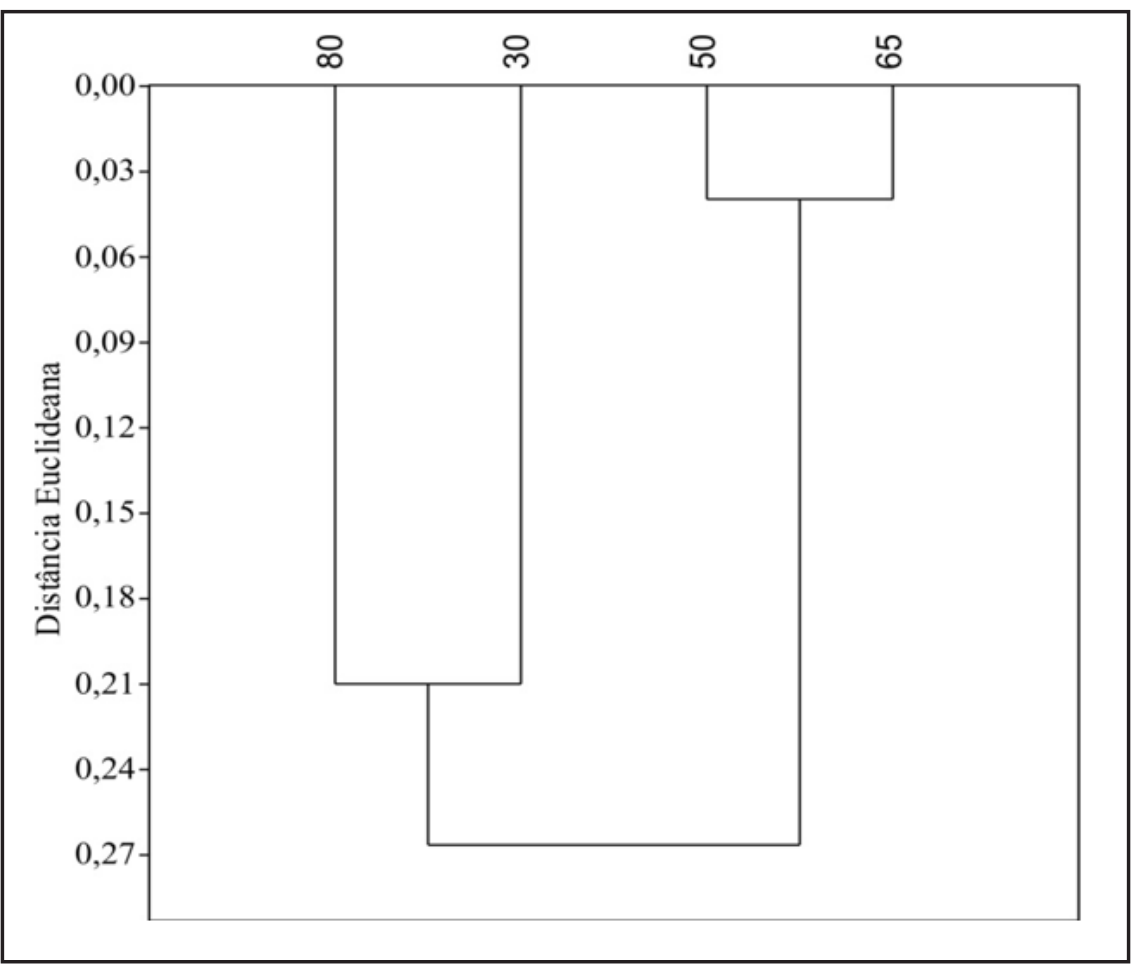

Fonte: Autores (2020) 


\section{CONCLUSÕES}

O plantio de leguminosas arbóreas fixadoras de nitrogênio é eficaz na ativação dos processos de sucessão ecológica, uma vez que garante um alto recrutamento de espécies nativas regenerantes, principalmente acima de 30\% da composição inicial. Em áreas como as do estudo, recomenda-se utilizar um percentual entre 50 e 65\% de espécies com essa capacidade na composição de plantio.

As espécies exóticas plantadas não foram encontradas no banco de plântulas regenerantes, com exceção de Pseudosamanea guachapele e Mimosa caesalpiniifolia. Cerca de 98\% dos indivíduos regenerantes não estavam na composição botânica do plantio inicial, tendo, portanto, origem nas áreas adjacentes, ou na ativação do banco de sementes de solo, a partir da cobertura florestal originada pelo plantio.

\section{REFERÊNCIAS}

AB'SÁBER, A. N. O domínio dos "mares de morros" no Brasil. Geomorfologia, São Paulo, n. 2, p. 1-9, 1966.

ALVES, T. G. Sobrevivência de espécies arbóreas nativas consorciadas à leguminosas nodulantes, Serra da Concórdia, Valença, Rio de Janeiro. 2007. Monografia (Bacharelado em Ciências Biológicas) - Universidade Severino Sombra, Vassouras, 2007.

CALDAS, A. J. F. da S. Geoprocessamento e análise ambiental para determinação de corredores de hábitat na Serra da Concórdia, Vale do Paraíba -RJ. 2006. Dissertação (Mestrado em Ciências Ambientais e Florestais) - Universidade Federal Rural do Rio de Janeiro, Seropédica, 2006.

CHAER, G. M.et al. Nitrogen-fixing legume tree species for the reclamation of severely degraded lands in Brazil. Tree Physiology, Oxford, v. 31, p. 139-149, 2011.

DERESZ, F. et al. Produção de leite de vacas Holandês x Zebu em pastagens de gramíneas tropicais manejadas sob pastejo rotativo. Juiz de Fora, MG: Embrapa Gado de Leite, 2006. 6 p. (Circular Técnica, 90).

FREITAS, W. K. de; MAGALHÃES, L. M. S. Métodos e parâmetros para estudo da vegetação com ênfase no estrato arbóreo. Floresta e Ambiente, Seropédica, v. 19, n. 4, p. 520-540, 2012.

FREITAS, W. K. de; MAGALHÃES, L. M. S. Florística, diversidade e distribuição espacial das espécies arbóreas de um trecho de Floresta Estacional Semidecidual da Serra da Concórdia, RJ. Floresta, Curitiba, v. 44, p. 259-270, 2014. 
IBGE. Manual técnico da vegetação brasileira. Rio de Janeiro: Fundação Instituto Brasileiro de Geografia e Estatística, Departamento de Recursos Naturais e Estudos Ambientais, 2012. 272 p. (Série Manuais Técnicos em Geociências, n. 1).

KELTY, M. J. The role of species mixtures in plantation forest. Forest Ecology and Management, Amsterdan, v. 233, p. 195-204, 2006.

LIMA, K. D. R.et al. Soil fauna as bioindicator of recovery of degraded areas in the caatinga biome. Revista Caatinga, Mossoró, v. 30, p. 401-411, 2017.

MACHADO, R. L.et al. Soil and nutrient losses in erosion gullies at different degrees of restoration. Revista Brasileira de Ciência do Solo, Viçosa, MG, v. 34, p. 945-954, 2010.

MARTIN, D. M. Ecological restoration should be redefined for the twentyfirst century. Restoration Ecology, Malden, v. 25, n. 5, p. 668-673, 2017.

MARTINS, E. M. et al. O uso de sistemas agroflorestais diversificados na restauração florestal na Mata Atlântica. Ciência Florestal, Santa Maria, v. 29, p. 632, 2019.

MELO, A. C. G.; DURIGAN, G. Evolução estrutural de reflorestamentos de restauração de matas ciliares no Médio Vale do Paranapanema. Scientia Forestalis, Piracicaba, v. 7, p. 101-111, 2007.

MENGE, D. N. L.; CHAZDON, R. L. Higher survival drives the success of nitrogen-fixing trees through succession in Costa Rican rainforests. New Phytologist, Cambridge, v. 209, p. 965977, 2016.

REFLORA. Herbário Virtual. Rio de Janeiro, [2015]. Disponível em: http://reflora.jbrj.gov.br/ jabot/herbarioVirtual/. Acesso em: 30 out. 2015.

RESENDE, A. S. et al. Uso de leguminosas arbóreas na recuperação de áreas degradadas. Tópicos em Ciência do Solo, Viçosa, MG, v. 8, p. 71-92, 2013.

RESENDE, A. S.; LELES, P. S. S. Controle de plantas daninhas em restauração florestal. 1. ed. Brasília, DF: Embrapa, 2017. 107 p.

RIBEIRO, M. C. et al. The Brazilian Atlantic Forest: How much is left, and how is the remaining forest distributed? Implications for conservation. Biological Conservation, Essex, v. 142, p. 1141-1153, 2009.

SANTANA, J. E. S. et al. Grasses Control Strategies in Setting Restoration Stand of the Atlantic Forest. Floresta e Ambiente, Seropédica, v. 27, n. 2, e20190066, 2020.

SANTOS, M. A. et al. Estratégias e ações para conservação da biodiversidade no Estado do Rio de Janeiro. In: BERGALLO, H. G. et al. Pressão antrópica e as novas dinâmicas na economia fluminense. Rio de Janeiro: Instituto Biomas, 2009. p. 41-65.

SILVA, A. B. et al. Estoque de serapilheira e fertilidade do solo em pastagem degradada de Brachiaria decumbens após implantação de leguminosas arbustivas e arbóreas forrageiras. Revista Brasileira de Ciência do Solo, Viçosa, MG, v. 37, p. 502-511, 2013. 


\section{Contribuição de Autoria}

\section{1 - Khadidja Dantas Rocha de Lima}

Engenheira Agrônoma, Dra., Pesquisadora

https://orcid.org/0000-0001-7575-0621•khadidjadantas@hotmail.com

Contribuição: Conceituação, Escrita - primeira redação

\section{2 - Maria Elizabeth Fernandes Correia}

Bióloga, Dra., Pesquisadora

https://orcid.org/0000-0003-1919-6659• elizabeth.correia@embrapa.br

Contribuição: Administração do projeto, Supervisão

\section{3 - Eduardo Francia Carneiro Campello}

Engenheiro Florestal, Dr, Pesquisador

https://orcid.org/0000-0003-4484-9738•eduardo.campello@embrapa.br

Contribuição: Obtenção de financiamento, Metodologia

\section{4 - Alexander Silva de Resende}

Engenheiro Florestal, Dr, Pesquisador

https://orcid.org/0000-0002-1433-9203• alexander.resende@embrapa.br Contribuição: Escrita - revisão e edição

\section{Como citar este artigo}

Lima, K. D.; Correia, M. E. F.; Campello, E. F. C.; Resende, A. S. Regeneração natural após 13 anos de plantio com espécies arbóreas fixadoras de nitrogênio em Valença, no estado do Rio de Janeiro Ciência Florestal, Santa Maria, v. 31, n. 2, p. 830-845, 2021. DOI 10.5902/1980509844460. Disponível em: https://doi.org/10.5902/1980509844460. Acesso em: xx mês-abreviado 2021. 